\title{
Acoustic emission as a tool for prediction of nugget diameter in resistance spot welding
}

\author{
Arnout Dejans ${ }^{\mathrm{a}, *}$, Oleksandr Kurtov ${ }^{\mathrm{a}}$, Patrick Van Rymenant ${ }^{\mathrm{a}}$ \\ ${ }^{a} K U$ Leuven, Jan Pieter De Nayerlaan 5, 2860 Sint-Katelijne-Waver, Belgium \\ https: //doi.org/10.1016/j. jmapro.2020.12.002
}

\begin{abstract}
Acoustic emission monitoring has been successfully employed in different application areas such as monitoring of fatigue loaded bridges and pressure testing of vessels. However, application of this technology on resistance (spot) welding processes yielded limited results. Existing literature only mentions the detection of certain defects such as expulsion and cracked welds. Detecting nugget nucleation and growth, to allow prediction of nugget diameter, has never been proven to be feasible based on acoustic emission signals. This work contains experiments based on varying as well as constant nominal welding parameter sets, where acoustic emission waveforms were collected during the welding process. A methodology is described, revealing a correlation between certain frequencies present in the acoustic emission signal and physical events occurring during welding. Based on these frequencies, a model is constructed for predicting the nugget diameter of resistance spot welds based on acoustic emission measurement data.
\end{abstract}

Keywords: acoustic emission, resistance spot welding, nugget diameter

\section{Introduction}

Resistance spot welding (RSW) is one of the resistance pressure welding processes that finds many applications in industry. Given that this process is highly automat5 able makes it one of the main joining methods for overlap joints of metallic, plate-like structures in mass production plants. The fact that a car and a flat panel household radiator respectively typically consist of 3500 and 5000 resistance spot welds underlines the importance of this welding 10 process.

Besides the follow-up of process parameters, the application of multiple in-line monitoring techniques on RSW processes have been researched. Some of these techniques showed promising results regarding in-line prediction of 15 weld nugget diameter.

To begin with, quality assessment based on the analysis of dynamic resistance measurements found its way to industry in the last decade and has been implemented in commercially available power sources, both as a quality 20 monitoring tool as well as to enable adaptive welding. Dynamic resistance based monitoring strategies rely on measurement of both the current and voltage of the secondary circuit of the welding machine, out of which an electrical resistance value is calculated for every moment in time.

25 Given that the most significant variation in resistance of the secondary circuit is caused by heating of the contact interface between the workpieces and the growth of a weld

\footnotetext{
* Corresponding author

Email address: arnout.dejans@kuleuven.be (Arnout Dejans)
}

nugget, the varying resistance can yield insight in the subsequent stages of the welding process. In early research [1], it was already proven that the calculated dynamic resistance of a RSW cycle allows to reveal the first point of melting, hence the nucleation of the welding nugget. The nugget diameter of the welded joint, often related to joint strength and thus an important measure for quality, can be extracted from the dynamic resistance data in two ways. On the one hand, methodologies have been developed based on calculations of the energy added to the melt. These calculations are conducted based on the calculated dynamic resistance and the measured welding current [2]. On the other hand, predictive models have been built based on the geometrical properties of the dynamic resistance curve [3]. Also, expulsion due to overheating was proven to be detected by dynamic resistance based monitoring systems [4], illustrating the wider range of application of this monitoring technique. A possible downside of these monitoring systems is the fact that they can only operate in the presence of a welding current, and thus cannot yield understanding in events happening after completion of the welding time, such as nugget solidification or the formation of cracks. Furthermore, it has been shown that a similar dynamic resistance signal does not always correspond to a comparable nugget diameter [5].

Besides, in-line quality assessment and estimation of nugget diameter in RSW has been performed based on ultrasound measurements. In contrast to the more conventional, off-line, variant of this quality assessment method, sensors are placed in the electrode caps. This allows for execution of the measurement during the full welding cycle, 
leading to measurements of nugget diameter and height

60 [6]. Recent optimisations of this approach allow for inline analysis of the measurement data, leading to commercially available implementations of this technique [7]. The major advantage of in-line ultrasound systems to-120 wards other in-line methodologies is that a geometrical urement takes place, whereas other monitoring techniques demand interpretation of measurements that are less obviously correlated to the weld's geometry. Downsides of these ultrasound-based systems are the cost and ${ }_{125}$ complexity added to the welding setup.

A third group of monitoring systems capable of estimating geometrical properties of a resistance spot welded nugget are those based on directly or indirectly measuring interactions between the workpieces and the welding ${ }_{130}$ electrodes. These interactions are caused by the expan-

75 sion and shrinkage of the base material when subjected to heating and cooling, in combination with deformations as a result of softening of the base material at elevated temperatures. They are quantified by measuring electrode ${ }_{135}$ force, electrode displacement or weld indentation. Far-

80 son [8] showed that electrode displacement curves could be correlated to both nugget height as well as nugget diameter. Xing [9] later applied displacement-based monitoring on shunted welds, and correlated displacement signal fea-140 tures to the final nugget diameter. The same dataset was 85 used to successfully correlate the final weld indentation and the dynamic resistance curves to the nugget diameter of these welds. Besides, monitoring techniques based on electrode force, electrode displacement or weld indentation ${ }_{145}$ have proven to be successful in recognizing expulsion [10].

As an alternative to the aforementioned monitoring techniques, acoustic emission (AE) could be implemented as an in-line, non-invasive quality monitoring system for RSW processes, possibly yielding further insight in weld ${ }_{150}$ formation and leading to non-destructive identification of nugget diameter. AE monitoring relies on the principle where part of the energy released during certain events escapes in the form of transient elastic waves. When these waves travel to the material's surface, the displacementsi55 they induce are measured by a piezoelectric sensor.

Analysis of AE signals is typically performed based on the detection of so called hits, based on which different properties are calculated. An AE hit is a part of the signal in the time domain which meets certain boundary condi-160 tions: a detection threshold, hit duration time, hit lockout time, peak definition time, etc. After extraction of these hits from the measured signal, their characterisation leads to properties such as their maximal amplitude, counts number of surpassing the detection threshold, energy and ${ }_{165}$ duration.

Hit-based analysis using these properties has been successfully applied to monitor processes and structures. Examples can be found in different fields, such as safety engineering, where fatigue crack initiation and growth could ${ }_{170}$ be monitored [11], and bearing condition monitoring [12]. in a relatively continuous signal that is present for a long time. In such cases, internal, structural changes could be accompanied by a distinct change in amplitude or peak frequency, making hit-based analysis a fast and cost-effective approach.

However, applications of $\mathrm{AE}$ as a quality monitoring technique in the field of RSW are not yet to be found in industry. One of the reasons might be the fact that existing AE monitoring systems are often hit-driven. As will be shown in this work, the welding current has a significant influence on the AE signal. It crowds out part of the AE events from the signal generated during the RSW process and makes hit-driven analysis of these events during welding troublesome, limiting the usage in an industrial environment.

Although existing research on AE monitoring of RSW processes is very limited, Yi [13] proved that certain characteristics of the RSW process, such as the welding current, could be recognized by AE characteristics. However, these process parameters can be monitored using conventional, much more (cost) effective methods. On the other hand, it was shown that a hit-driven approach of AE signals yields insight in splashing during the RSW process and the occurrence of cracks during and after solidification $[13,14]$.

A potential additional benefit of $\mathrm{AE}$ monitoring in the field of RSW could be the in-line, non-invasive measurement of the nugget diameter during the welding process. The nugget diameter of a spot weld is often one of the key requirements related to its quality and strength. Despite multiple attempts to estimate the diameter of spot welds by means of AE measurements, there is still no procedure available which allows correlating the acquired AE signal to physical events, such as nugget nucleation and nugget growth, that take place during the welding process. Previous researches have led to conclusions about weld diameter or joint strength based on processing AE signals in the time domain. Yi et al. [13] showed the influence of weld nugget diameter on AE hit counts and hit amplitude during RSW of $2 \mathrm{~mm} 2024$ aluminium alloy plates. The results of this research show a linear increasing relationship between counts as well as hit amplitude and the weld nugget diameter. Besides, Podržaj [15] demonstrates a correlation between the shear tensile strength of welds and the extracted AE features. RSW experiments were carried out on $1 \mathrm{~mm}$ zinc coated steel. AE characteristics, such as counts, were defined as predictors for the shear tensile strength of the welds. In this case, the acquisition of sonic emission was done by means of a microphone.

Both studies discussed above have drawn conclusions based on datasets generated with altered welding parameters, such as welding current and time. Based on their data, it is impossible to prove that observations in the $\mathrm{AE}$ signals were caused by nugget formation and its growth, rather than by the welding current.

Current literature attempted defining nugget nucleation and growth based on AE monitoring using hit-based anal- 


\begin{tabular}{ccccc}
\hline no. & $\begin{array}{c}\text { current } \\
(\mathrm{kA})\end{array}$ & $\begin{array}{c}\text { force } \\
(\mathrm{N})\end{array}$ & $\begin{array}{c}\text { time } \\
(\mathrm{ms})\end{array}$ & $\begin{array}{c}\text { nominal nugget } \\
\text { diameter } \\
(\mathrm{mm})\end{array}$ \\
\hline \hline 1 & 6.0 & 2500 & 70 & 2.22 \\
2 & 7.2 & 2500 & 70 & 3.98 \\
\hline
\end{tabular}

Table 1: Overview of parameter sets (machine settings) used in this study.

ysis. The goal of this work is to observe nugget nucleation and predict the nugget diameter during RSW by applying an alternative approach and performing signal analysis in the frequency domain.

\section{Experimental setup}

Experiments described in this work are performed on an ARO servo-driven RSW machine with a $1000 \mathrm{~Hz}, 90 \mathrm{kVA}$ DC power source, depicted in figure 1 . The machine is equipped with water cooled electrode caps with an ISO 5821:2009 FE-15.8-5.5-30 geometry. New electrode caps were used for each experiment. Electrodes were not cleaned in between welds.

Two experiments were conducted using machine parameters as summarized in table 1. For each experiment, 50 realisations were made. This replicates a limited run in a production setup, where only (unexpected) environmental changes are present and nominal welding parameters carbon steel plates with dimensions of 20 by $50 \mathrm{~mm}$ and a thickness of $1 \mathrm{~mm}$. Samples were welded in as-delivered conditions, meaning no cleaning or degreasing was performed.

The AE signals were detected during welding by a Mistras wideband sensor, having an optimal frequency range of 125 to $1000 \mathrm{kHz}$ and a resonant frequency of $450 \mathrm{kHz}$. The signals were amplified by a Mistras preamplifier using a $40 \mathrm{~dB}$ gain. The preamplified data was acquired by a pling rate of $2 \mathrm{MHz}$. Simultaneously, the data acquisition system recorded the electrical current in the welding circuit, measured by a PEMRGF $300 \mathrm{~S}$ Rogowski coil and preamplifier. Finally, the temperature at both electrode thermocouples were positioned on the side surface of the electrode caps, $15 \mathrm{~mm}$ from the electrode tip. All signals were acquired during a period of $100 \mathrm{~ms}$, starting $5 \mathrm{~ms}$ before the application of the welding current, as schematically represented in figure 3 . Signal processing was executed in Matlab.

The AE sensor was placed on the bottom electrode holder of the RSW machine, as shown in figure 2. This reduces the effect the servo controlled actuator, that drives22 the top electrode holder, could have on the measured signals. Silicon based grease was used as a couplant to provide good contact between sensor and electrode holder.

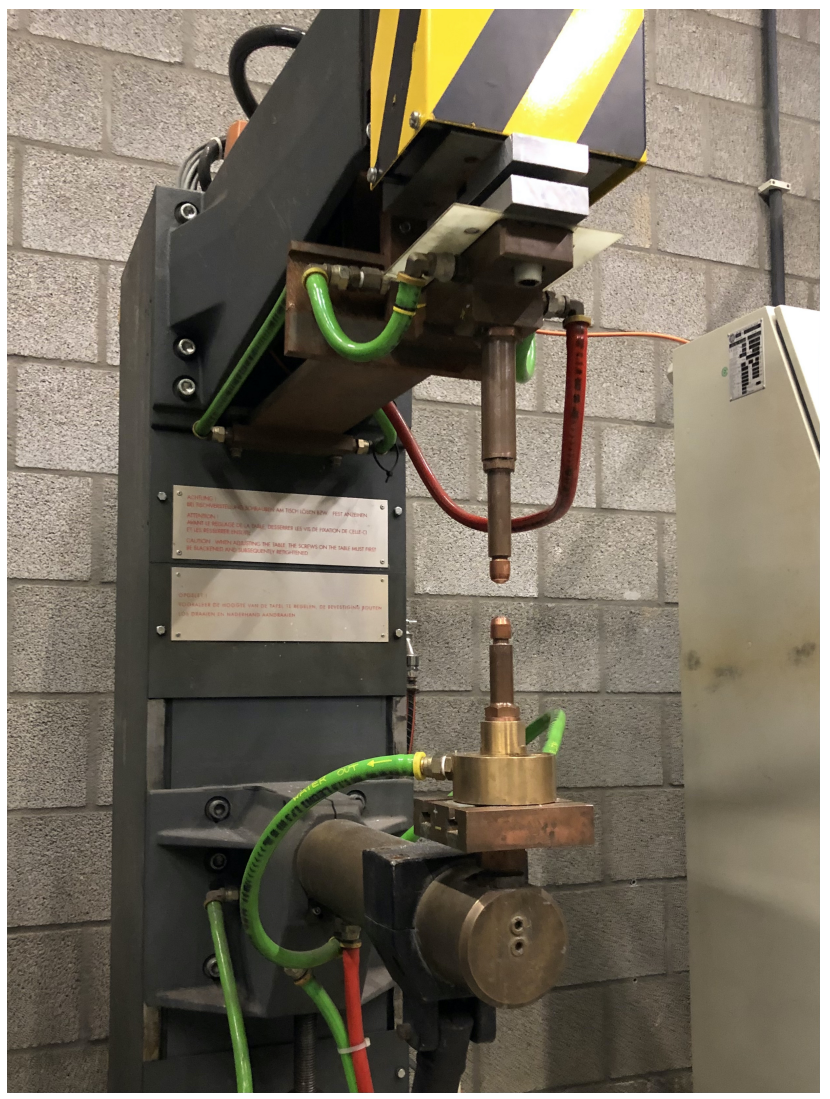

Figure 1: Setup used during this study, consisting of a servo-driven ARO resistance welding machine with a MFDC power source.

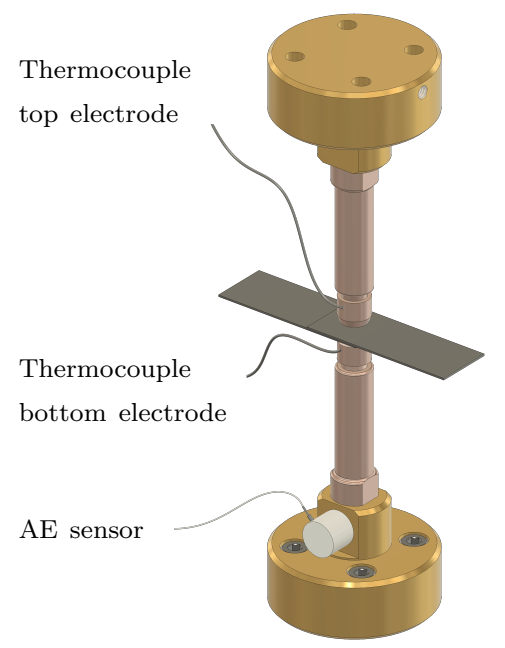

Figure 2: Schematical representation of the AE sensor positioned on the bottom electrode holder, as well as the thermocouples on the electrode caps.

By deliberately not placing the sensor on one of the workpieces, the measurements mimic an industrial situation in a more realistic way, where constant installation and removal of the sensor on a workpiece would be impossible given the production flow in a mass production plant where this joining process finds its applications. 


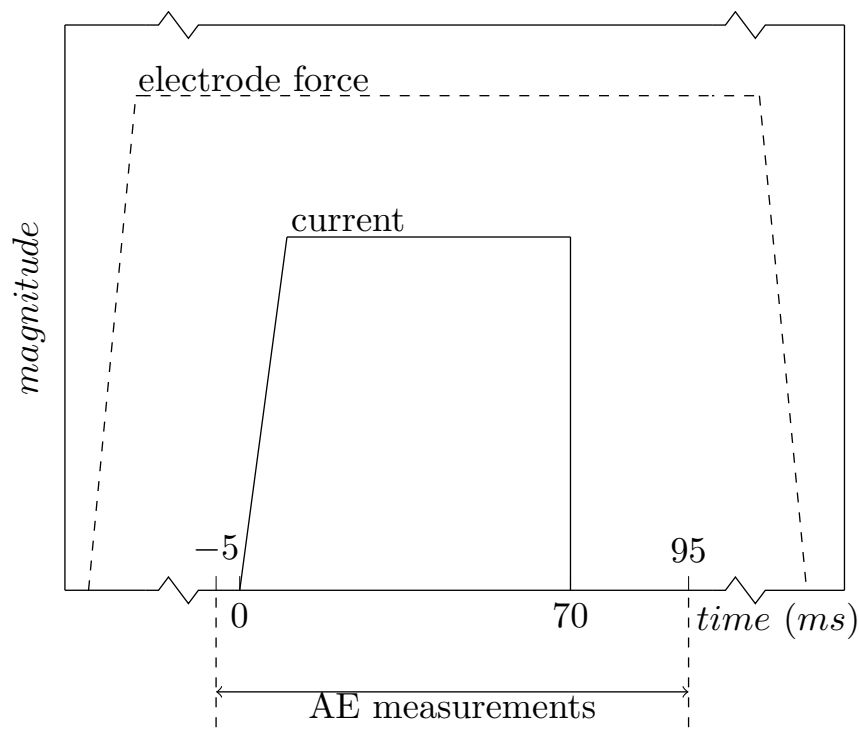

Figure 3: Schematical representation of the moment of signal acquisition, relative to the start of the welding current.

The acquired sensor data is compared with physical measurements. Therefore, all weld nugget diameters were measured using calipers according to ISO 10447:2015. Parameter sets 1 and 2 respectively resulted in a nominal nugget diameter of $2.22 \mathrm{~mm}$ and $3.98 \mathrm{~mm}$. All samples of parameter set 1 had a nugget diameter smaller than $.5 \mathrm{~mm}$, whilst all welds made with parameter set 2 resulted in nugget diameters larger than this value. The diameter of $3.5 \mathrm{~mm}$ corresponds to the lower limit of the welding lobe diagram according to ISO 14327:2004, 3.5 $\sqrt{t}$, where $t$ is the thickness of a single plate. Hence, this is the minimal required nugget diameter according to this standard. Lap shear tests were performed using an Instron 4206 system.

\section{Signal analysis and results}

The nugget diameters, although constant machine parameters were used, differ significantly within one parameter set. For parameter set 1, 9 of the 50 welds resulted in a stuck weld (no nugget formation) or a weld of which ${ }^{260}$ the nugget diameter was too small to repeatably measure. The reason, as illustrated in table 2 and elaborated below, is the low welding current and time compared to a robust parameter set used in production. As the goal of this work is to correlate the diameter of the molten nugget265 to the acquired AE signal, and as these samples don't have a nugget to correlate to, these samples were not taken into account in this study. All joints produced with parameter set 2 resulted in a molten nugget and are included in this analysis.

Figure 4 proves the differences in nugget diameter cannot be attributed to variations in welding current or initial electrode temperature, and are thus not to be detected
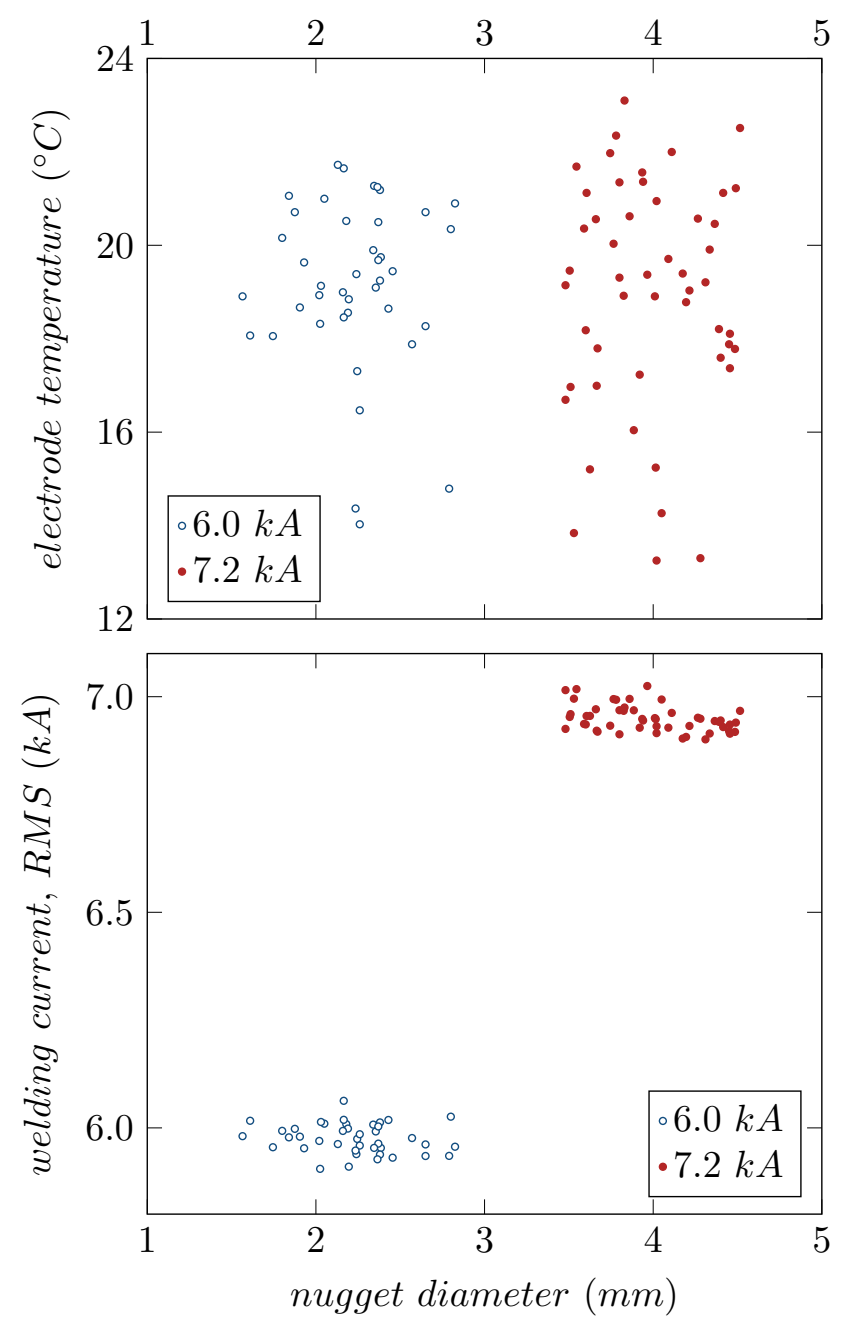

Figure 4: Nugget diameters for samples of parameter set 1 and 2, compared to the electrode temperature at the start of the welding time (top) as well as to the RMS of the measured welding current (bottom).

by conventional measurements of welding parameters during the RSW process. It should be noted that this variation in nugget diameter for constant machine parameters is not preferred in a production environment, but was deliberately induced by selecting welding parameters with a lower welding time, compared to one that would be typically chosen in production.

The latter is visualised in table 2, depicting the nominal nugget diameter as well as its standard deviation for welds made using an electrode force of $2500 \mathrm{~N}$, a welding current of $6.0 \mathrm{kA}$ and $7.2 \mathrm{kA}$ and four different welding times. The data in this table is generated based on 5 samples for each parameter combination. Increasing the welding time results in two observations. On the one hand, welds with a longer welding time result in a larger nugget diameter. However, the rate of growth in nugget diameter slows down with increasing welding time. When too much heat is added to the joint, and expulsion occurs, the weld nugget diameter could slightly decrease in diameter as a 
equence of the expelled metal. On the other hand, the standard deviation on the nugget diameter decreases with increasing welding time. This is a consequence of the decreasing rate in nugget growth, diminishing the effect ${ }_{325}$ of small changes in the moment of nugget nucleation or ${ }_{280}$ heat generation between individual welds in one dataset. Selecting a parameter combination with a relatively short welding time $(70 \mathrm{~ms})$ thus allows for studying differences in nugget diameter without taking the effects of varying welding current on the AE signal into account.

\begin{tabular}{c|cccc}
\hline current & $35 \mathrm{~ms}$ & $70 \mathrm{~ms}$ & $110 \mathrm{~ms}$ & $130 \mathrm{~ms}$ \\
\hline \hline $6.0 \mathrm{kA}$ & $0.00 \pm 0.00$ & $2.22 \pm 0.30$ & $3.94 \pm 0.17$ & $4.33 \pm 0.10$ \\
$7.2 \mathrm{kA}$ & $0.00 \pm 0.00$ & $3.98 \pm 0.32$ & $5.08 \pm 0.24$ & $4.90^{*} \pm 0.13$
\end{tabular}

Table 2: The effect of welding time on nominal nugget diameter ${ }^{335}$ $(\mathrm{mm})$ and standard deviation on this value. Asterisk denotes the occurrence of expulsion.

\subsection{Signal analysis using acoustic emission hits}

Subjecting the AE measurement data of samples welded ${ }^{40}$ with these parameter sets to hit-based analysis in the time domain did not yield any information about nugget formation. The differences in hit properties (such as amplitude, energy, etc.) could solely be related to the difference in welding current applied. It was not possible to detect the ${ }^{345}$ moment of nucleation, neither predict the nugget diameter, based on this hit-based analysis.

A potential reason can be found in the large influence trates that the amplitude of the AE signal is highly de-350 pendent on the welding current applied. On the one hand, this figure shows the course of the AE signal for a sample from both parameter set 1 and 2, visually indicating a difference in amplitude of these signals. On the other hand, this figure depicts two enlarged windows of these ${ }^{355}$ signals around the $30^{\text {th }}$ millisecond. As earlier shown in table 2, no welding joint is present at this moment in time. Hence, comparing the AE signal of both parameter sets of a welding nugget are taken into account. Consequently, ${ }^{360}$ the differences in the AE signal at the $30^{\text {th }}$ millisecond can solely be attributed to the difference in welding current. The amplitude's RMS value, peak value and the counts The average values of these properties over all samples in ${ }^{365}$ each parameter set are summarised figure 5 as well. An increased amplitude can be observed for the parameter set using a higher welding current. This has a significant im-

315 pact on hit-based analysis techniques, where the start and the end of a hit is characterized by crossing a threshold ${ }^{370}$ level.

Hence, one can conclude that the the results of hitbased analysis will be highly dependent on the magnitude of the welding current. However, as figure 4 illustrated earlier, the variation in welding current within one parameter ${ }^{375}$ set is no sufficient predictor for explaining the variance in nugget diameter. Consequently, the influence the welding current has on the AE signal, is not beneficial for estimating the nugget diameter. The authors believe that, although the metallurgical phenomena emit transient waves, their influence is crowded out by the effects of the welding current when hit-based analysis in the time domain is performed.

\subsection{Signal analysis using frequency analysis}

As an alternative on hit-based analysis in the time domain, a methodology is presented revealing frequencies occurring in the AE signal which can be correlated to the final nugget diameter of the weld. Before demonstrating the potential of such a characteristic frequency for predicting the nugget diameter of a resistance spot weld, its evolution will be compared to physical phenomena occurring during welding.

\subsubsection{Selection of characteristic frequencies}

As stated earlier, it is hypothesized that different physical phenomena taking place during welding, such as the presence of molten material, are accompanied with an increase or decrease in amplitude of acoustic transient waves at a certain frequency present in the measurement signal. In order to validate this theory and find a relationship between the presence of certain frequencies in the AE signal and the final nugget diameter of a weld, the frequency response of the AE measurement signal is analyzed at different moments during the welding cycle. These are later correlated with the nugget diameter of the welds. The different steps used to find these correlations, hence the characteristic frequencies to be used for prediction of nugget diameter, are schematically visualized in the flowchart in figure 6 and explained below.

The first step of this analysis consists of dividing the AE measurement signal into $1 \mathrm{~ms}$ segments. The goal of implementing this segmentation prior to executing the frequency analysis is twofold. On the one hand, a certain time resolution is maintained, allowing localisation of the detected physical events relative to the start of the welding time. On the other hand, phenomena that only have a limited influence on the AE signal, either in amplitude or in time, have a higher chance of standing out in a segmented signal compared to a single frequency analysis performed over the full length of the AE signal.

In the second step, a frequency analysis is performed. In the case of this study, this was implemented by applying Goertzel's algorithm for one specific frequency on each segment of the signal. This filter calculates a single bin discrete Fourier transform in a computationally efficient way, given that only the frequency response of a scarce amount of frequency bins is of interest [16]. This computational efficiency is essential for potential implementation of in-line analysis of AE signals in a production environment, where both computational power and time are more 

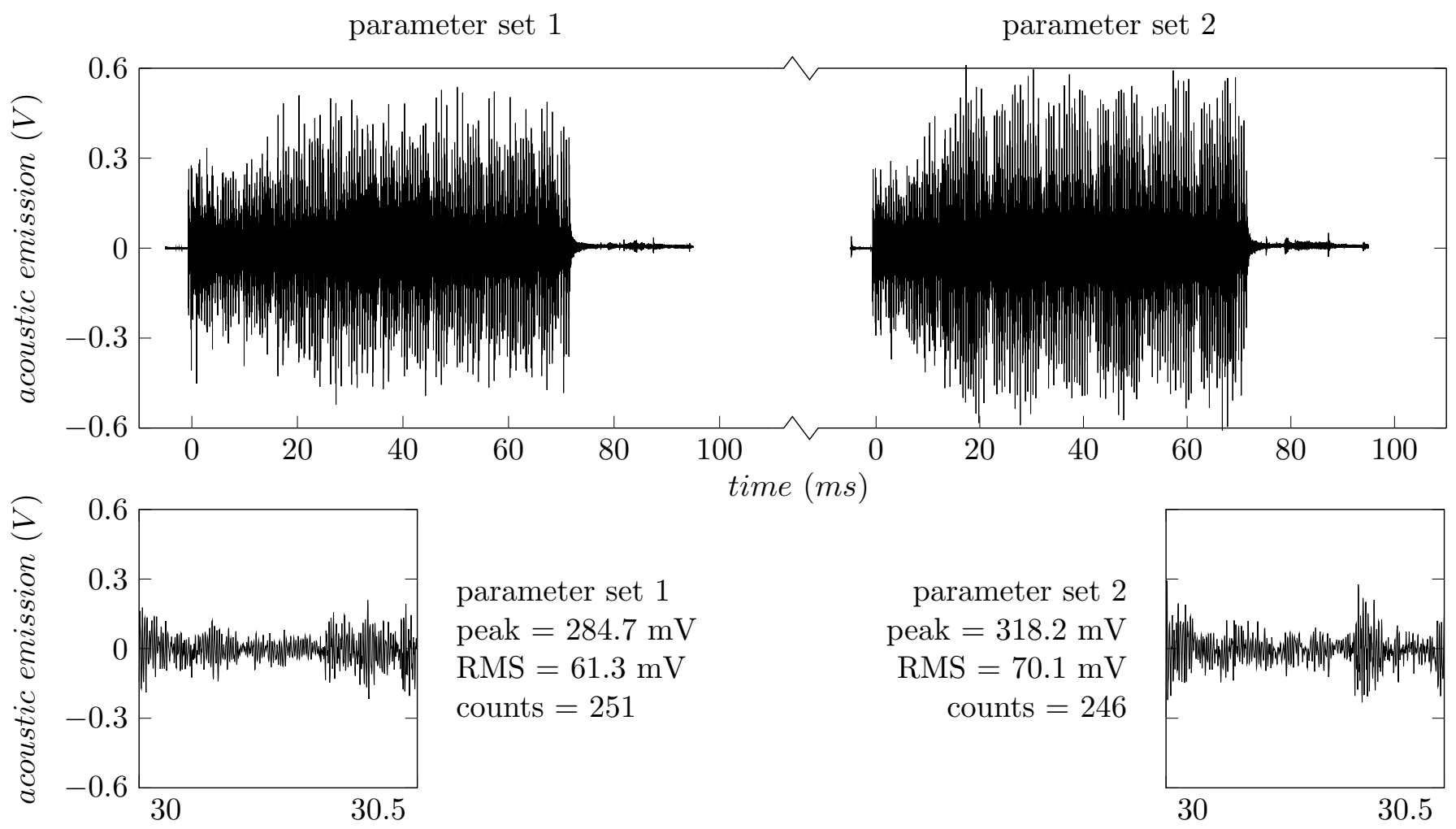

parameter set 1

peak $=284.7 \mathrm{mV}$

$\mathrm{RMS}=61.3 \mathrm{mV}$

counts $=251$ parameter set 2

peak $=318.2 \mathrm{mV}$

$\mathrm{RMS}=70.1 \mathrm{mV}$

counts $=246$

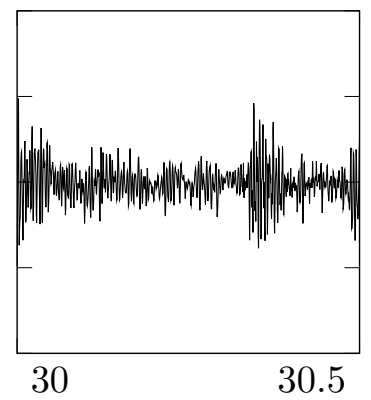

Figure 5: Top: Comparison of the AE waveforms captured for welds made with the two welding parameter sets described in table 1. Bottom: Enlarged windows at $30 \mathrm{~ms}$ demonstrating the influence of the welding current on the AE signal. Calculated properties are the average over the full parameter set.

limited compared to a lab setup. In the presented analysis, Goertzel's algorithm was applied using the Matlab function goertzel, based on [17]. When using Goertzel's405 algorithm, the width of the frequency bin is equal to the between the sampling rate and the length of the segment studied. Given a sampling rate of $2 \mathrm{MHz}$ and a sample size of 2000, the number of samples in one segment of $1 \mathrm{~ms}$, the frequency bins are $1000 \mathrm{~Hz}$ wide. It should be 410 clear that the segment width selected in the first step of of the analysed signal, but also influences the frequency resolution.

The third step of the analysis procedure consists of 415 revealing possible correlations between the calculated frequency responses and the measured final nugget diameter of the welded samples. Simple linear regression models are composed at every segment for which the frequency response was calculated. The coefficient of determination ${ }_{420}$ $\left(\mathrm{r}^{2}\right)$ is used as a measure for the goodness of fit for these models.

This three step procedure is repeated for different frequency bins, ranging from $1 \mathrm{kHz}$ to $1 \mathrm{MHz}$, resulting in an overview of frequencies present in the $\mathrm{AE}$ measurement signal which are correlated to the final nugget diameter ${ }_{425}$ of the resistance welded joint. This procedure was performed separately for welds made with parameter set 1 and 2 . The results are graphically represented in figure 7 by plotting the coefficients of determination for the linear relationships between the frequency response of varying frequencies and the final nugget size at varying times. For the sake of clarity, this plot only contains frequencies in a range between $1 \mathrm{kHz}$ and $150 \mathrm{kHz}$, as this range contained the highest coefficients of determination.

It can be noticed that the coefficients of determination are generally lower for the welds in parameter set 1 , compared to these resulting from parameter set 2 . The highest coefficient of determination over both experiments was to be found at a frequency of $52 \mathrm{kHz}$ in the case of parameter set $2\left(\mathrm{r}^{2}=0.62\right)$. For parameter set 1 , that same frequency resulted in a coefficient of determination of $\mathrm{r}^{2}=0.43$. The only other frequency that results in a relatively high coefficient of determination for both parameter sets is $46 \mathrm{kHz}$, where a maximum of $\mathrm{r}^{2}=0.49$ and $\mathrm{r}^{2}=0.52$ occurs for parameter set 1 and 2 respectively. For these reasons, both $46 \mathrm{kHz}$ and $52 \mathrm{kHz}$ will be investigated as characteristic frequencies for prediction of nugget diameter based on the AE measurement signal in the current welding setup.

\subsubsection{Physical explanation of the correlation values}

In order to study how the correlations between the amplitude of these frequencies and the final nugget diameter evolve during the welding time, and to be able to link these correlations to physical phenomena occurring during welding, the correlation values are compared to the 


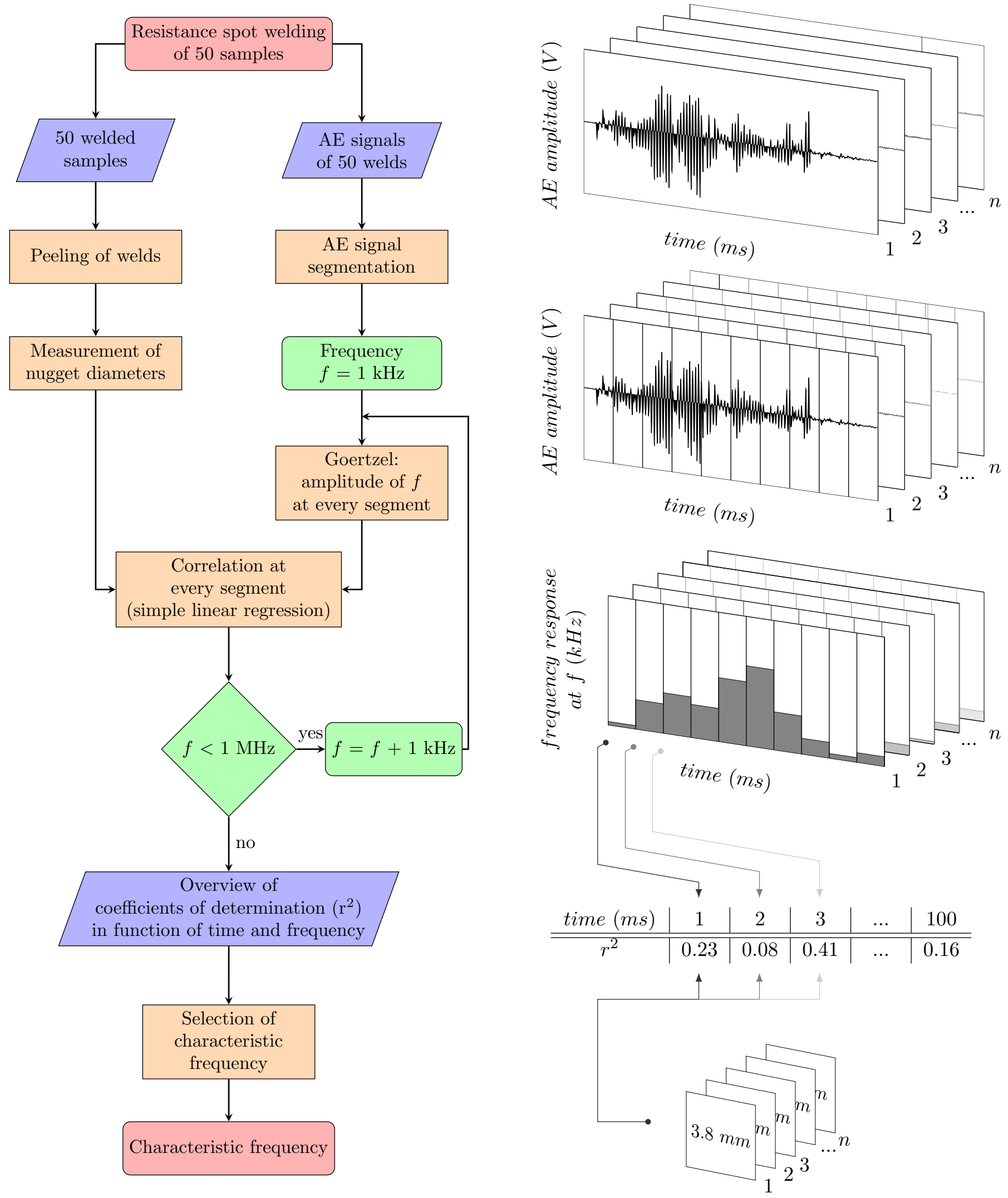

Figure 6: Left: Flowchart of the steps taken in order to determine a characteristic frequency which contains information with regard to nugget diameter. Right: Schematical visualisation of the most important elements present in the flow chart. From top to bottom: AE signals; segmented AE signals; frequency response at certain frequency for all segments; correlation of frequency response to nugget diameters. 
parameter set 1

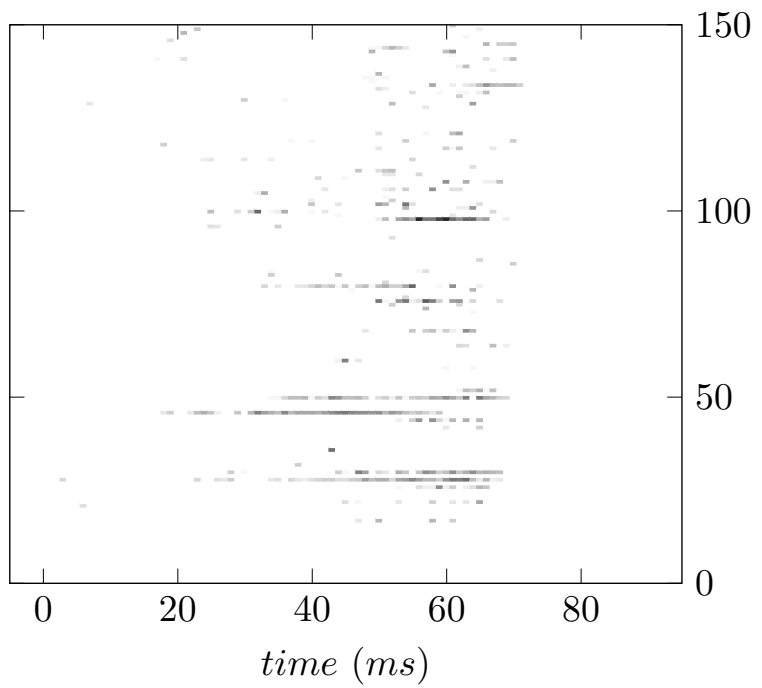

parameter set 2

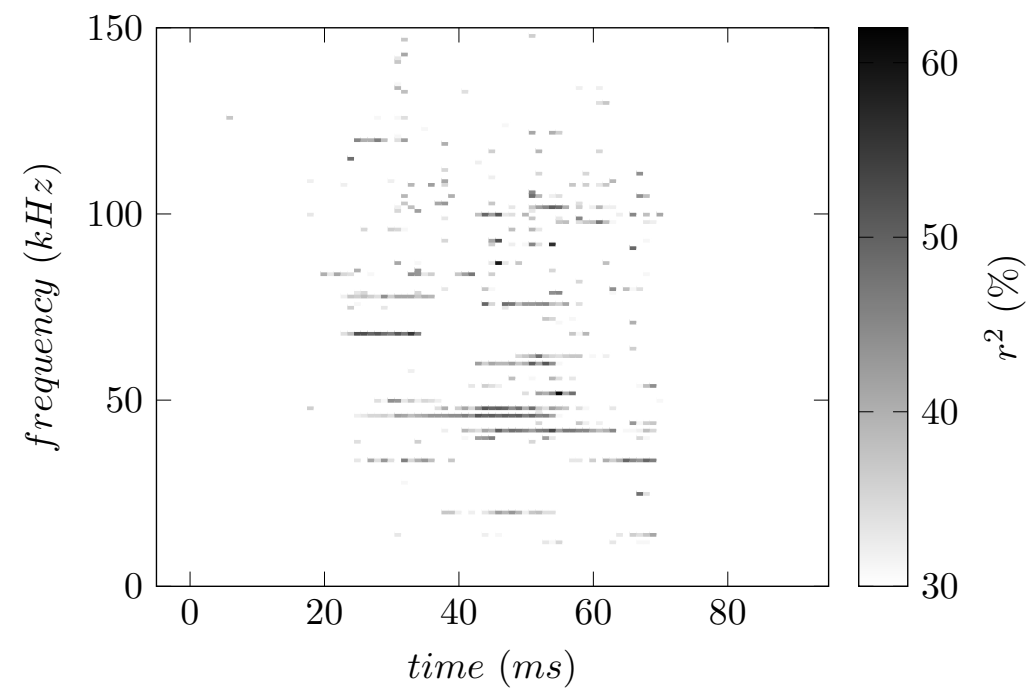

Figure 7: Overview of coefficients of determination for relationships between amplitude of a given frequency and final nugget diameter, calculated for varying frequencies and varying moments during the welding cycle.

fracture surfaces and tensile shear strength of welds made with varying welding time. Although the welds made in dataset 1 and 2 are based on a welding time of $70 \mathrm{~ms}, 465$ welding times for this experiment range from $0 \mathrm{~ms}$ up to $125 \mathrm{~ms}$. This yields an overview of the evolution in shear tensile strength, as well as the standard deviation on this value, for welds made with increasing welding time. Figure 8 depicts these fracture surfaces, tensile shear strengths470 and correlation values for both parameter combinations, welded with a welding current of $6.0 \mathrm{kA}$ and $7.2 \mathrm{kA}$ respectively. The correlation values are plotted for both frequencies of $46 \mathrm{kHz}$ and $52 \mathrm{kHz}$, selected based on the analysis in the previous section.

The fracture surfaces, resulting from tensile shear loading, of welds produced with varying welding times were studied by optical microscopy. For each parameter set, figure 8 contains four pictures of these fracture surfaces where respectively no bond, a corona bond, a beginning ${ }_{480}$ nugget and a nugget are present. These fracture surfaces indicate that, nominally, nucleation occurred around $62 \mathrm{~ms}$ and $55 \mathrm{~ms}$ for parameter set 1 and 2 respectively. However, as slight variation in contact conditions, heating, etc. will cause this value to vary between successive welds with ${ }_{485}$ identical parameters, these values cannot be captured exactly. Hence, these observations should be used as indicative rather than exact values.

The shear tensile strength values plotted in figure 8 are the result of welds produced with constant electrode ${ }_{490}$ force and welding current, but where different welding times were applied. Therefore, the plots contain the mean value of 5 replicates for each welding time, while the error bars depict the standard deviation of these measurements. Three important observations can be made. Firstly, the 495 shear tensile strength of the welds stabilises after a weld- ing time of $100 \mathrm{~ms}$ and $80 \mathrm{~ms}$ for parameter set 1 and 2 respectively. Secondly, lower welding times are accompanied with a higher scatter in shear tensile strength. These two observations illustrate why the parameter sets presented in table 1 are accompanied with a great uncertainty in nugget size, as noticed earlier and plotted in figure 4 . The welding times of parameter set 1 and 2 are selected in a region where the weld nugget is still growing and did not yet reach its final size and strength. Hence, slight variations, for example in contact conditions, will cause nugget diameters to differ more significantly compared to a parameter set with an increased welding time, where the shear tensile strength stabilised. Thirdly, one can observe that the shear tensile strength rapidly increases around a welding time of $62 \mathrm{~ms}$ and $55 \mathrm{~ms}$ for parameter set 1 and 2 respectively. This, combined with the observations made based on the fracture surfaces, proves that a nugget nucleated around this time. The shear strength achieved for welds produced with a lower welding time can be attributed to the presence of the brazed-like corona bond.

The lower part of figure 8 depicts the evolution of the correlation between final nugget diameter and the frequency response at the frequencies found in the section above: $46 \mathrm{kHz}$ and $52 \mathrm{kHz}$. For both of these frequencies, the evolution in the coefficient of determination can be linked to the evolution in shear strength and the fracture surfaces studied after this test.

Given the $52 \mathrm{kHz}$ frequency bin, no relationship is present between the final nugget diameter and the amplitude at this frequency in the beginning of the welding phase. This statement can be made for both parameter sets. The scatter in correlation values present in the first $20 \mathrm{~ms}$ of the welding time could be attributed to outliers in amplitude at the $52 \mathrm{kHz}$ frequency rather than a consistent corre- 
lation. The presence of these outliers in the beginning of the welding time could potentially be explained by the 555 fact that the welding current generates an electromagnetic field, proportional with the magnitude of the welding current, around the conductors it flows through. At the beginning of the welding cycle, the welding current rises rapidly. As a consequence, the corresponding electromagnetic field ${ }_{560}$ and the forces it exerts on surrounding objects, grow accordingly. The fact that the magnetic force is applied over a relatively short time, results in vibrations in the subjected objects, on their turn leading to acoustic emission being captured by the sensors in the setup. A similar ef-565 fect can be observed at the moment the welding current rapidly slopes down.

A linear relationship starts to grow between the amplitude at the frequency bin of $52 \mathrm{kHz}$ and the nugget diameter after approximately $50 \mathrm{~ms}$ and $38 \mathrm{~ms}$ for parameter 570 set 1 and 2 respectively, as can be deducted from the inhappens earlier than the nominal moment of nucleation, experimentally determined based on fracture surfaces and shear tensile strengths (62 ms and $55 \mathrm{~ms}$ for parameter set 1 and 2 respectively), shows that the correlation at this fre- 575 quency is not caused by the presence of a molten nugget. Further investigation shows that the formation of a corona bond starts around $50 \mathrm{~ms}$ and $38 \mathrm{~ms}$ respectively, as is also revealed by the slightly increasing shear strength for welds made with this welding time and the corresponding frac- ${ }_{580}$ ture surface, both depicted in figure 8 .

Although the welding current ends after $70 \mathrm{~ms}$, and the weld nugget should thus still be present until that moment, at the $62^{\text {th }}$ (parameter set 1) and $55^{\text {th }}$ millisecond (parameter set 2) of the welding time, the correlation reaches $a_{585}$ ximum at the given frequency. After this moment, the correlation decreases.

At a frequency bin of $46 \mathrm{kHz}$, the coefficients of determination for the linear relationship between the amplitude at this frequency and the final nugget diameter follow $a_{590}$ Starting from the moment the welding current is applied, the coefficient of determination rises consistently. This phenomenon is observed for both parameter sets. A maximum is reached after 45 and $43 \mathrm{~ms}$ for parameter set 1 and 2 respectively, whereafter the $\mathrm{co}^{-}{ }_{595}$ 540 efficient of determination declines and reaches a quasi-zero value when the welding time terminates. Linking these observations to the physical phenomena observed in the shear tensile strengths as well as the fracture surfaces leads to two major conclusions. Firstly, the fact that the coefficient ${ }_{600}$ 545 of determination starts rising before joining phenomena such as a corona-bond or melting occur implies that the growth in coefficient of determination has to be attributed to based on AE waves generated by other phenomena, such as burning of impurities at the contact surface. Secondly,605 the peak in coefficient of determination for the analysis based on the $46 \mathrm{kHz}$ frequency bin is not to be consistently attributed to a certain physical event deduced from the fracture surfaces or shear strength data. In the case of parameter set 1 , this maximum occurs around the moment of corona bond formation. While for parameter set 2, the peak in coefficient of determination is located between the moment of corona bond formation and the moment of nucleation. A potential reason could be the varying moments of nucleation (and corona bond formation) for welds within one parameter set, making it impossible to link the maximum value to the nominally observed moment of nucleation (and corona bond formation). The fact that the data based on the $46 \mathrm{kHz}$ frequency bin seems more difficult to be correlated to the physical phenomena observed makes it a less trustworthy source for nugget diameter prediction. Nevertheless, the consistency of the results suggests that further investigation, possibly by a different technique is appropriate.

It is important to note that the processed AE signal is correlated to the final nugget diameter, after solidification. The setup used does not allow for in-situ, non invasive measurements nugget diameter measurement, during the welding process.

\subsubsection{Prediction of nugget diameter}

In the sections above, it was described how linear relationships between nugget diameter and the amplitude at a certain frequency in the AE signal were evaluated. Besides, for two frequencies, the linear relationship between these quantities was further investigated and linked to physical phenomena occurring during welding. Based on evaluation of the amplitude of these frequencies present at specific moments in the AE measurement signal, it is thus possible predicting the nugget diameter of a weld in a nondestructive way.

Figure 9 summarises how the predicted nugget diameter is evaluated for both parameter set 1 (left) and 2 (right), based on the characteristic frequency of either $46 \mathrm{kHz}$ (top) or $52 \mathrm{kHz}$ (bottom). For each of these four situations, figure 9 depicts the following. On the one hand, it shows the relationship between the predicted nugget diameter and the measured nugget diameter for the welds performed in each dataset. This prediction is made based on the amplitude at either a frequency of $46 \mathrm{kHz}$ or $52 \mathrm{kHz}$. The graph depicting this relationship shows the predicted nugget diameter on the left y-axis, whilst the amplitude at the characteristic frequency on which this prediction is based, is shown on the right y-axis. On the other hand, the figure shows the evolution of the amplitude at this characteristic frequency for the welds resulting in the smallest and the largest nugget diameter of each dataset. On that same graph, a vertical line denotes the moment based on which the nugget diameter estimation is performed. As was mentioned in section 3.2.2, and depicted in figure 8, this time value is depending on the parameter set as well as the characteristic frequency used for the nugget diameter prediction.

For the characteristic frequencies of $46 \mathrm{kHz}$ and $52 \mathrm{kHz}$, the predictions of parameter set 1 result in a root mean 
parameter set 1

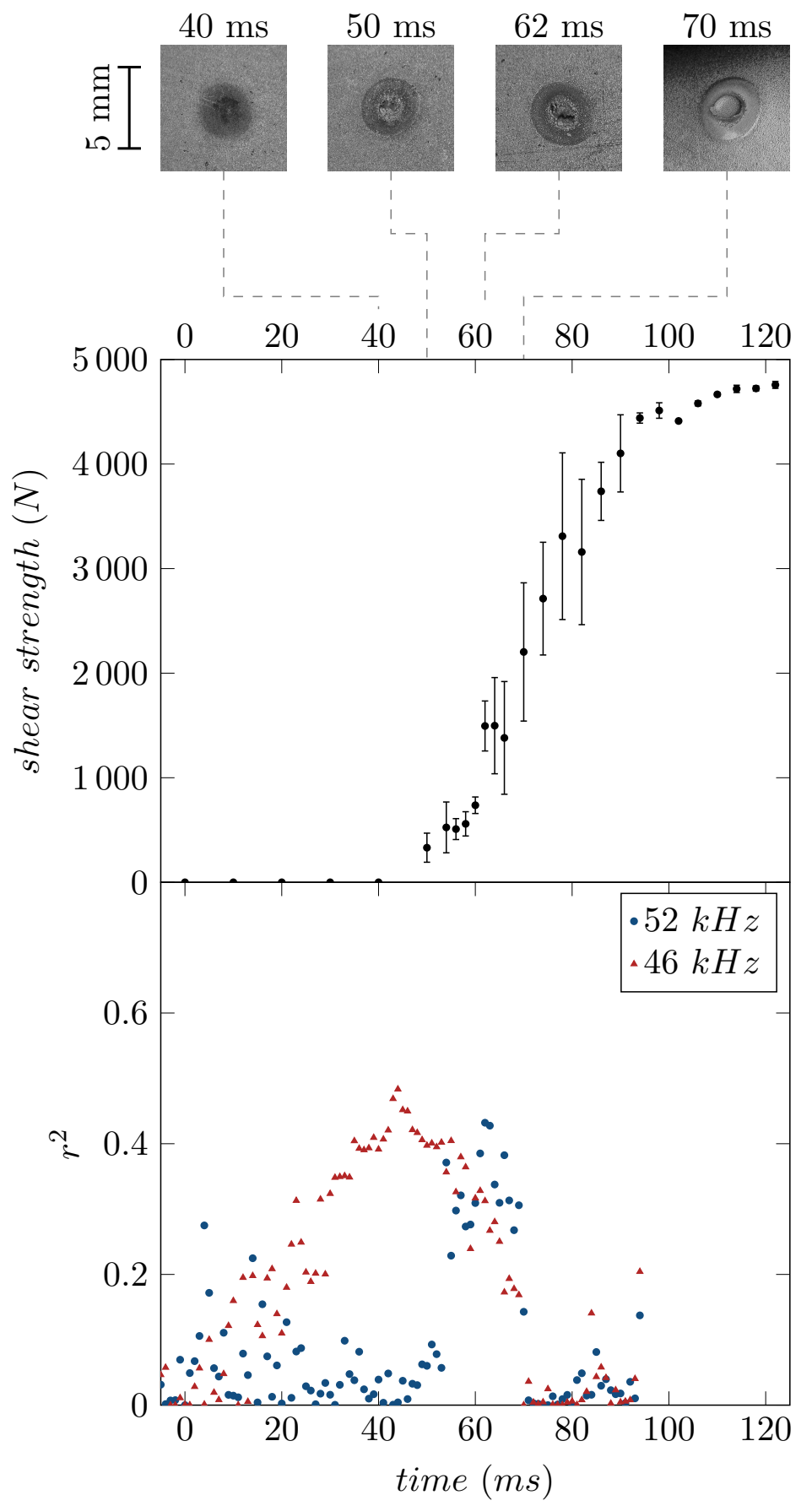

parameter set 2

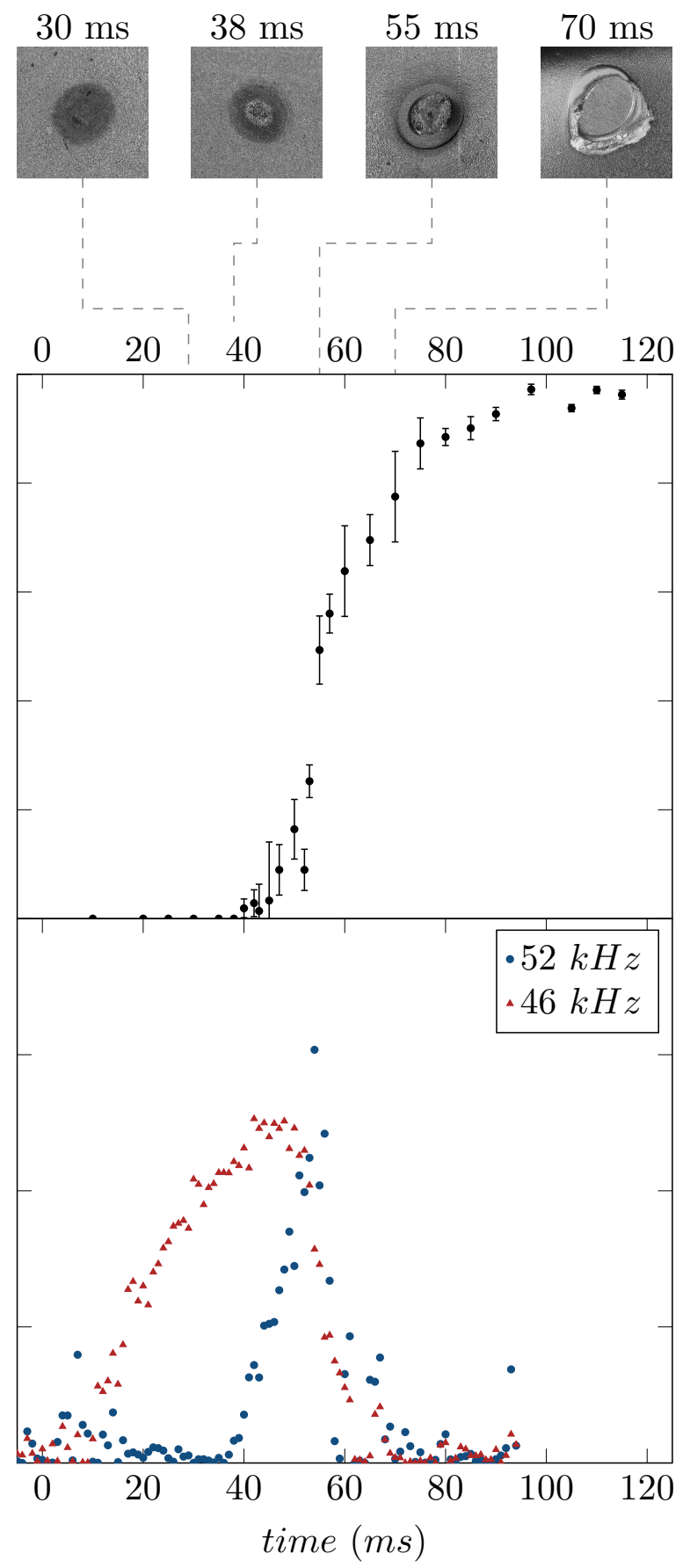

Figure 8: Top: Fracture surfaces of lap shear tensile tested spot welds produced with varying welding times. Center: Lap shear strength of spot welds produced with varying welding times. Error bars depict standard deviation of the measurement. Bottom: Coefficient of determination for the linear relationship between measured final nugget diameter and the amplitude at the frequency bin of $46 \mathrm{kHz}$ and $52 \mathrm{kHz}$ at different moments in time. Information plotted for parameter set 1 (left) and 2 (right).

square error (RMSE) of $0.33 \mathrm{~mm}$ and $0.37 \mathrm{~mm}$. The predictions of parameter set 2 result in a RMSE of $0.33 \mathrm{~mm}$ and $0.27 \mathrm{~mm}$ for these frequencies respectively.

\section{Discussion}

Hit-based analysis performed on AE signals from welds of both parameter sets revealed that the welding current 615 signal, hence on the detection of a hit. As a result, eval- 

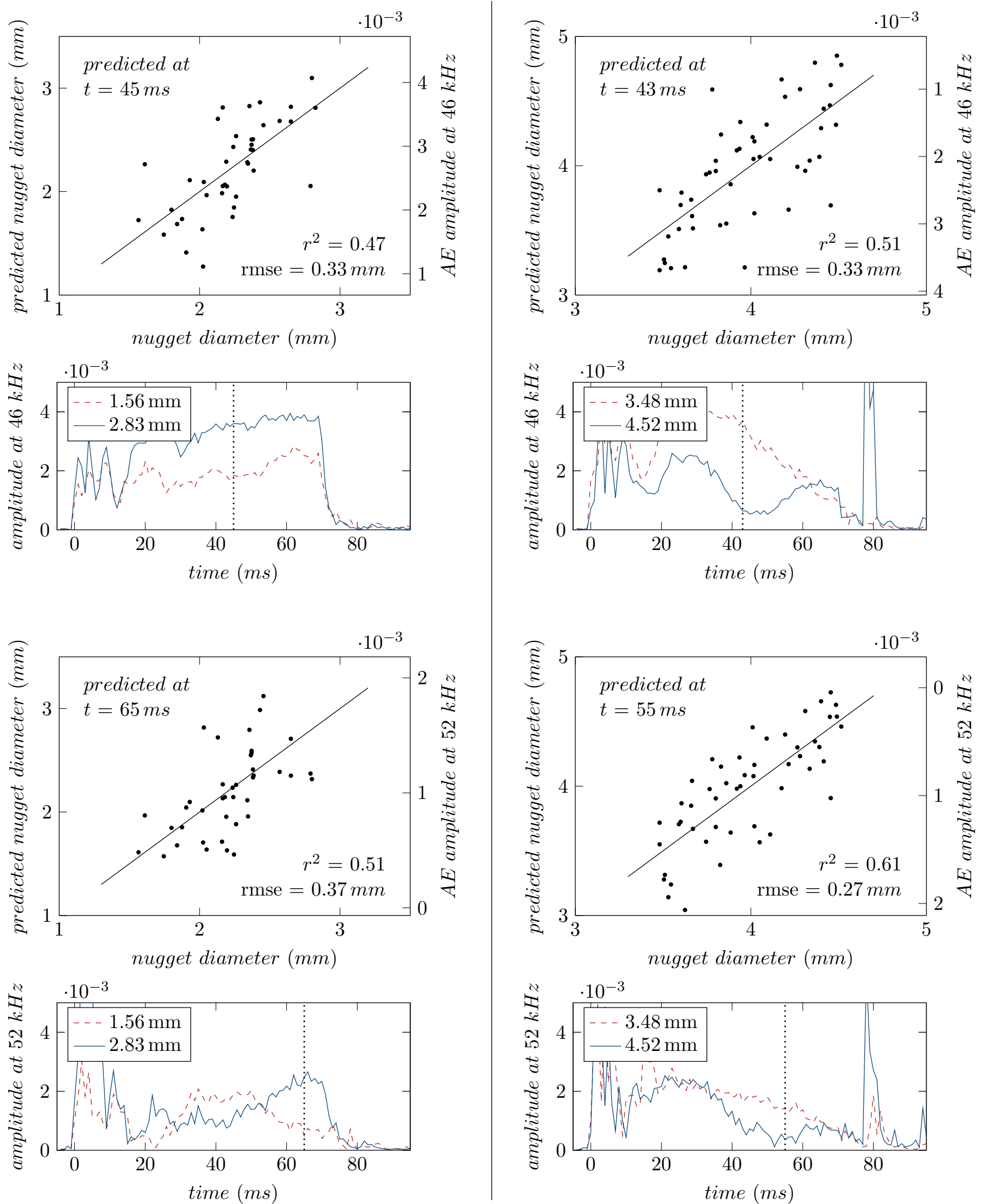

Figure 9: Plots for combinations of parameter set 1 (left) and 2 (right) with a characteristic frequency of $46 \mathrm{kHz}$ (top) and $52 \mathrm{kHz}$ (bottom). Above: Relationship between the amplitude at a characteristic frequency (as well as the derived predicted nugget diameter) and the nugget diameter. Below: Amplitude of a characteristic frequency in function of time, plotted for the smallest and largest weld in the dataset. A vertical line depicts the moment in time on which the nugget prediction is based. 
uating the quality or nugget diameter of welds made with different welding parameters subjected to the same hit-675 based analysis, as described in [13] and [18], is arguable as it is not proven that the features detected are to be attributed to phenomena such as nugget growth rather than welding current itself. These observations further indicate the importance of developing alternative approaches of $\mathrm{AE}_{680}$ measurement signals analysis for evaluating different quality related aspects of resistance welds.

The frequency-based methodology presented in this work proves the potential of using certain frequencies present in the AE measurement signal for estimating the final nugget ${ }_{685}$ diameter of a RSW joint. Important to note is that, although the observed amplitudes in the AE signal differed for different welding parameter sets, the same two frequencies could be correlated to the final diameter of the joint. However, the magnitude of the selected characteristic fre-690 quencies is not consistent between the two studied paramin figure 9. For example, for parameter set 1, the amplitude is higher at the $46 \mathrm{kHz}$ frequency bin for larger joints, whilst the opposite is true for parameter set 2. This illus-695 trates the importance of developing a separate prediction model for each parameter set.

When linking physical phenomena such as the presence of a corona bond and nugget nucleation to the amplitude of the selected characteristic frequencies, it seems to be im-700 possible to evaluate the diameter of the nugget during the correlations drop to a minimum value before the welding current ends. However, a clear relationship was found between the amplitude of the selected characteristic frequen-705 cies and the final nugget diameter of the joint before the nugget was formed. The measurements leading to these predictions are made before the experimentally determined moment of nucleation, but during the presence of a corona bond. This strengthens the assumption that it is 710 the brazed-like corona bond that causes interpretable $\mathrm{AE}$ 655 signals which are proportional to the final nugget diameter. As depicted in figure 8, the evolution of the fracture surfaces allows to assume that gradual changes in surface conditions, such as burning of impurities and the oxide 715 layer, in the presence of electrical current and electrode

${ }_{660}$ force cause audible noise. A similar hypothesis was made by Podržaj [15], but his work did not support this assumption with physical observations.

The reason for the sudden drop in the coefficient of $f_{720}$ determination, close to the nominal time where nugget 665 nucleation takes place, is not easily attributed to a single event. One possibility is that the increasing growth of the nugget shifts the characteristic frequency to different values. The liquid melt pool could act like a damper for ${ }_{725}$ the frequencies generated in the area of the corona bond.

670 Another possibility is that this drop in correlation is not to be attributed to a physical event. Alternatively, it could be caused by the methodology used. As can be seen in figure 9 , the course of the magnitude at the selected fre-730 quencies could be characterised by certain features (such as peaks, slopes, etc.). Possibly, correlating these features to the weld diameter yields better results compared to the absolute amplitudes presented in this work. Further research should investigate these hypotheses.

Based on the correlations between nugget diameter and specific characteristic frequencies, predictions of the nugget diameter were made. Depending on the machine settings and the characteristic frequency used, a RMSE between 0.27 and $0.37 \mathrm{~mm}$ was achieved, using a simple linear regression model. Criticism could arise pointing that these values are relatively high, hence not suitable for trustworthy prediction of nugget diameters. However, three aspects should be taken into account.

Firstly, it is important to note that the physical measurements of the nugget diameter, performed according to ISO 10447:2015, include an error related to the measuring method. The weld nuggets are not perfectly round, causing the measurements of the nugget diameter to differ depending on the measurement location. Besides, the calipers used for the measurement are typically too thick to reach the nugget and measure it in a reliable and repeatable way, causing a systematic error on the measurement. These measurement errors when measuring the nugget diameter of resistance spot welds have been thoroughly described by [19]. For more accurate analysis, further experiments could thus include the application of different measuring techniques.

Secondly, comparing these results with existing, hitbased, literature on AE-based quality monitoring is not possible, as no results of nugget diameter prediction based on a dataset generated with constant machine parameters are reported. The latter is important to be able to differentiate between the effects of machine parameters and weld nugget diameter.

Thirdly, the predictions reported in this work are based on one predictor, in other words, a single characteristic frequency. Although the prediction errors of the proposed AE-based methodology are higher compared to those reported for dynamic-resistance based methodologies (e.g. RMSE $=0.06 \mathrm{~mm}$ as published by Zhang [20]), these estimations are based on multiple features of the dynamic resistance signal. The AE-based methodology could be further optimised by combining the response of multiple characteristic frequencies. Instead of the physical approach presented in this work, further research could apply machine leaning techniques in order to extract multiple predictors from the AE signal, and combining these predictors in a more robust model for nugget diameter prediction.

The linear regression models presented in this work, predicting nugget diameter based on a measured AE signal, are highly dependent on the welding parameters used. This can be seen from the data presented in figure 9. The slope of the studied relationship does not only differ in magnitude depending on the welding parameters used, the direction of the slope is also reversed between the models constructed for dataset 1 or 2 . This dependency of the 
model parameters on welding process parameters has a785 large influence on the universality of the predictive model, as (possibly minor) changes in process parameters will demand for construction of a new prediction model. The latter is a time consuming task, making this sensitivity to process parameters a significant downside of the developed ${ }_{790}$ methodology. The experiments performed in this work did not allow to unambiguously define the root cause for this phenomenon.

As mentioned in section 3, 9 samples of parameter set 1 were excluded for the analysis. Reason being that these samples happened to result in stuck welds. Consequently, correlating the AE signal of these welds to the studied physical measurements was impossible. Although the decribed analysis proved nugget diameter prediction based on AE monitoring to be feasible, the authors were not able to apply the proposed methodology to classify the ${ }_{800}$ stuck welds. The absence of a welding nugget could have an influence on the frequency response of the AE signal, independent from the diameter of the nugget. Hence, a different methodology could be needed for classification based on presence of a nugget compared to the methodology followed for developing the prediction model for nugget ${ }^{805}$ diameter estimation. Further research based on a larger dataset with a higher number of unexpected stuck welds could lead to a methodology to detect this phenomenon.

This work proves the potential of AE measurement signals to be applied for predicting the nugget diameter of re- ${ }_{810}$ sistance spot welds. However, further improvements in the setup and methodology should be made in order to lower the prediction error. The currently used methodology uses Goertzel's algorithm, as its computational efficiency could allow for in-line implementation. However, the frequency resolution of the methodology as implemented here is pos-815 sibly not satisfactory and could cause loss of important data. Alternative analysis techniques, such as wavelet analysis, could potentially lead to more accurate results. Besides, the work presented here aimed at selecting frequencies that were applicable on both parameter sets used. potentially be achieved by combining different characteristic frequencies for each parameter set. Finally, the current $_{820}$ methodology links absolute amplitudes of a characteristic frequency at a certain moment in time to the nugget diamA different approach, where relative changes in this value over time are linked to the nugget diameter could potentially lead to improved results. Such approaches are ${ }^{825}$ successfully applied to both dynamic resistance as well as electrode displacement signals [9].

\section{Conclusions}

This paper describes a methodology for revealing linear relationships between nugget diameter and the amplitude ${ }_{835}$ at a certain frequency in the AE signal. This methodology was applied on two experiments, each containing welds produced with constant machine parameters. Physical phenomena, such as the formation of a corona bond and the presence of a weld nugget were correlated to characteristic frequencies. Besides, the presence of these characteristic frequencies in the captured AE signal was used to predict the nugget diameter of welds. The conclusions can be summarized as follows:

- It was proven that the machine parameters, i.e. the welding current, have a significant influence on the acquired AE signal. It is thus impossible assessing the weld's characteristics with one single method for welds produced with different parameters.

- Due to the limited effect of nugget nucleation and growth on the AE signal, classical, hit-based AE signal analysis is not capable of predicting differences in nugget diameter for welds made with identical machine parameters.

- Based on a frequency analysis of the AE signal, in this case by applying Goertzel's algorithm at $46 \mathrm{kHz}$ and $52 \mathrm{kHz}$, physical phenomena such as the moment of corona bond formation and nugget nucleation could be linked to the AE measurement signal.

- The results of this frequency analysis could be correlated with the final nugget diameter of the spot weld, making it possible to predict the diameter of the joint based on the AE signal.

- This work proves the potential of nugget diameter prediction by AE monitoring: predictions are made with an RMSE up to $0.27 \mathrm{~mm}$. Further improvements are proposed in order to increase the prediction accuracy and develop an industrially applicable AE monitoring system.

\section{Acknowledgements}

This work was funded by Flanders Innovation \& Entrepreneurship (VLAIO no. HBC.2017.0241).

\section{References}

[1] D. Dickinson, J. Franklin, A. Stanya, et al., "Characterization of spot welding behavior by dynamic electrical parameter monitoring," Weld. J., vol. 59, no. 6, p. 170, 1980.

[2] K. Zhou and L. Cai, "Online nugget diameter control system for resistance spot welding," The Int. J. of Adv. Manuf. Technol., vol. 68, pp. 2571-2588, Mar 2013.

[3] X. Wan, Y. Wang, D. Zhao, Y. Huang, and Z. Yin, "Weld quality monitoring research in small scale resistance spot welding by dynamic resistance and neural network," Measurement, vol. 99, pp. $120-127,2017$.

[4] Y.-J. Xia, Z.-W. Su, Y.-B. Li, L. Zhou, and Y. Shen, "Online quantitative evaluation of expulsion in resistance spot welding," J. of Manuf. Process., vol. 46, pp. 34-43, 2019.

[5] L. Zhou, Y.-J. Xia, Y. Shen, A. S. Haselhuhn, D. M. Wegner, Y.B. Li, and B. E. Carlson, "Comparative study on resistance and displacement based adaptive output tracking control strategies for resistance spot welding," Journal of Manufacturing Processes, 2020. 
[6] A. Chertov, A. Karloff, W. Perez, A. Lui, and R. G. Maev, "In-process ultrasound nde of resistance spot welds," InsightnonDestr. Test. and Cond. Monit., vol. 54, no. 5, pp. 257-261, 2012.

[7] A. Baradarani, L. M. Khanli, A. Chertov, W. Regalado, and R. G. Maev, "Efficient feature extraction in ultrasonic spot weld inspection," in 2017 IEEE 30th Can. Conf. on Electr. and Comp. Eng. (CCECE), pp. 1-4, IEEE, 2017.

[8] D. Farson, J. Chen, K. Ely and, and T. Frech, "Monitoring resistance spot nugget size by electrode displacement," J. Manuf. Sci. Eng., vol. 126, no. 2, pp. 391-394, 2004.

[9] B. Xing, Y. Xiao, and Q. H. Qin, "Characteristics of shunting effect in resistance spot welding in mild steel based on electrode displacement," Meas., vol. 115, pp. 233-242, 2018.

[10] S. Chen, T. Sun, X. Jiang, J. Qi, and R. Zeng, "Online monitoring and evaluation of the weld quality of resistance spot welded titanium alloy," J. of Manuf. Process., vol. 23, pp. 183-191, 2016.

[11] G. N. Morscher and R. Maxwell, "Monitoring tensile fatigue crack growth and fiber failure around a notch in laminate sic/sic composites utilizing acoustic emission, electrical resistance, and digital image correlation," J. of the Eur. Ceram. Soc., vol. 39, no. 2 , pp. 229-239, 2019

[12] S. Poddar and N. Tandon, "Detection of particle contamination in journal bearing using acoustic emission and vibration monitoring techniques," Tribol. Int., vol. 134, pp. 154-164, 2019.

[13] Y. Luo, J. Li, and W. Wu, "Characterization of nugget nucleation quality based on the structure-borne acoustic emission signals detected during resistance spot welding process," Meas., vol. 46, no. 3, pp. 1053-1060, 2013.

[14] L. Yi, W. Rui, X. Xiaojian, and Z. Yang, "Expulsion analysis of resistance spot welding on zinc-coated steel by detection of structure-borne acoustic emission signals," The Int. J. of Adv. Manuf. Technol., vol. 84, no. 9-12, pp. 1995-2002, 2016.

15] P. Podržaj, I. Polajnar, J. Diaci, and Z. Kariž, "Estimating the strength of resistance spot welds based on sonic emission," Sci. and Technol. of Weld. and Join., vol. 10, no. 4, pp. 399-405, 2005.

[16] G. Goertzel, "An algorithm for the evaluation of finite trigonometric series," The Am. Math. Mon., vol. 65, no. 1, pp. 34-35, 1958.

880 [17] C. S. Burrus and T. Parks, DFT/FFT and Convolution Algorithms. Wiley, 1985.

18] P. Podržaj, I. Polajnar, J. Diaci, and Z. Kariž, "Expulsion detection system for resistance spot welding based on a neural network," Meas. Sci. and Technol., vol. 15, no. 3, p. 592, 2004.

[19] F. Almeida, T. De Paula, R. Leite, G. Gomes, J. Gomes, A. Paiva, and P. Balestrassi, "A multivariate gr\&r approach to variability evaluation of measuring instruments in resistance spot welding process," J. of Manuf. Process., vol. 36, pp. 465479, 2018.

[20] H. Zhang, Y. Hou, T. Yang, Q. Zhang, and J. Zhao, "Welding quality evaluation of resistance spot welding using the timevarying inductive reactance signal," Meas. Sci. and Technol., vol. 29 , no. 5 , pp. $1-13,2018$ 